\title{
Urine YKL-40 versus Urine NGAL as Potential Markers for Diagnosis of Urinary Tract Infection in Febrile Pediatric Patients
}

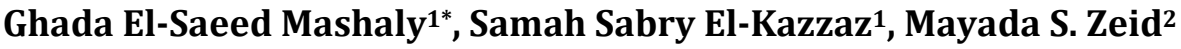 \\ ${ }^{1}$ Medical Microbiology and Immunology Department, Faculty of Medicine, Mansoura University, Mansoura, Egypt \\ ${ }^{2}$ Pediatrics Department, Faculty of Medicine, Mansoura University, Mansoura, Egypt \\ Email: ^ghadamashaly@yahoo.com
}

How to cite this paper: El-Saeed Mashaly, G., El-Kazzaz, S.S. and Zeid, M.S. (2020) Urine YKL-40 versus Urine NGAL as Potential Markers for Diagnosis of Urinary Tract Infection in Febrile Pediatric Patients. Open Journal of Immunology, 10, 10-20.

https://doi.org/10.4236/oji.2020.101002

Received: January 3, 2020

Accepted: March 21, 2020

Published: March 24, 2020

Copyright $\odot 2020$ by author(s) and Scientific Research Publishing Inc. This work is licensed under the Creative Commons Attribution International License (CC BY 4.0).

http://creativecommons.org/licenses/by/4.0/

(c) (i) Open Access

\begin{abstract}
Early diagnosis of urinary tract infection (UTI) in pediatric patients is a problem due to the absence of specific symptoms and difficulty in obtaining the proper urine sample. These difficulties increase the need for effective biomarker for UTI diagnosis in young patients. Neutrophil gelatinase-associated lipocalin (NGAL) is proved to be a marker for UTI diagnosis. YKL-40 is a glycosyl hydrolase which is produced locally at the sites of inflammation. The objective of our study is to assess the value of YKL-40 as a possible marker for UTI diagnosis in febrile children and to compare its value versus NGAL. Urine culture was used as a gold standard for UTI diagnosis. The study enrolled three groups; febrile children with positive urine culture, febrile children with negative urine culture, and controls without fever and with negative urine culture. Each group included 50 children from patients attended outpatients clinics department of Mansoura University Children Hospital. For each patient enrolled in the study, complete blood count, C-reactive protein, serum creatinine, urine creatinine, routine urine analysis and urine culture were assessed. Using ELISA test, urine values of NGAL (uNGAL) and YKL-40 (uYKL-40) were measured and normalized to urine creatinine ( $\mathrm{uNGAL} / \mathrm{uCr}$ ) and (uYKL40/uCr) respectively. The values of uNGAL, uYKL-40, uNGAL/Cr and $\mathrm{uYKL}-40 / \mathrm{Cr}$ were significantly higher in febrile UTI group. The receiver operating curve (ROC) show the optimum cut off value for urine YKL-40 (171.5 pg) with $84 \%$ senstivity, $82 \%$ specificity and the area under the ROC curve (AUC) 0.95 . The optimum cutoff value of uYKL-40/Cr was (159.2 $\mathrm{pg} / \mathrm{mg}$ ) with $72 \%$ sensitivity, $71 \%$ specificity and 0.81 AUC. Higher sensitivity and specificity of uYKL- 40 and uYKL40/Cr compared to uNGAL and uN$\mathrm{GAL} / \mathrm{Cr}$ respectively were observed. In conclusion, the diagnostic value of uYKL-40 is superior to that of UNGAL. Urine YKL- 40 could be a good marker for diagnosis of UTI in febrile pediatric patients.
\end{abstract}


Keywords

UTI, YKL-40, NGAL, Fever, Pediatrics, Biomarker

\section{Introduction}

Urinary tract infection (UTI) is a common infection in pediatric patients [1]. The standard method for diagnosis is urine culture. However, culture requires at least 2 days for confirmation of diagnosis. Delay in diagnosis of UTI may result in renal involvement and chronic renal damage. So, early diagnosis of UTI is essentially important to avoid renal complications [2] [3]. Therefore, the evaluation of new biomarkers for early diagnosis of UTI in pediatric patients is required [4] [5].

YKL-40 or Chitinase 3-Like Protein 1 (CHI3L1) is a member of the glycosyl hydrolase family 18 . The name of YKL-40 is derived from the NH2-terminal amino acids tyrosine $(\mathrm{Y})$, lysine $(\mathrm{K})$, and leucine $(\mathrm{L})$ and its $40 \mathrm{kDa}$ molecular weight [6] [7].

Various cells, including immune cells (e.g.) neutrophils and macrophages express YKL-40. YKL-40 is strongly induced during the late stages of human macrophage differentiation [8]. YKL-40 possesses a functional carbohydrate-binding motif but it lacks enzymatic activity entirely. It is not yet known to have a specific receptor [9] [10]. The exact biological function of YKL-40 is still undetermined. It can play a role in many processes like extracellular matrix, fibrosis, and inflammation [7]. YKL-40 could be a site-specific inflammatory marker because it is produced locally at inflammatory sites [11] [12].

Many biomarkers have been investigated for their role in diagnosing UTI. One of these markers is neutrophil gelatinase-associated lipocalin (NGAL). In innate immune response to Gram-negative bacteria, NGAL plays an important role. It binds to iron-laden bacterial siderophores preventing the absorption of iron and hence the growth of bacteria. NGAL functions as a neutrophil chemoattractor. It enhances neutrophil maturation, adhesion, phagocytosis, and bacterial killing. NGAL is expressed in healthy people with very low levels in neutrophils and epithelial cells of many organs like kidneys and liver [2] [13]. Urine NGAL (uNGAL) assay can be used for diagnosing UTI in children [4] [14] [15].

Few studies search the possible use of urineYKL-40 (uYKL-40) as a marker for UTI diagnosis in children. Our study aims at the assessment of uYKL-40 as a biomarker for UTI in pediatrics and compares this value versus uNGAL.

\section{Methods}

This study is a case control study which enrolled 150 children (aged between 1 month to 16 years) during period extending from February 2016 to March 2018. Patients attended the outpatient clinics of Mansoura University Children Hospital. 


\subsection{Inclusion Criteria}

Patients were classified into three groups each includes 50 patients:

Group (1): febrile children diagnosed with UTI based on clinical and lab criteria.

- Clinically presented with fever $\left(\geq 37.5^{\circ} \mathrm{C}\right)$, and symptoms suggesting UTI as back pain, difficult painful micturation, change in urine colour as stated from patient or mother.

- Lab criteria: positive urine culture with one organism $\geq 10^{5}$ colony-forming units $(\mathrm{CFU}) / \mathrm{mL}$.

- Urine samples were collected as early as possible prior to the start of the symptoms.

Group (2): febrile children without UTI.

- Clinically presented with fever $\left(\geq 37.5^{\circ} \mathrm{C}\right)$ and no clinical symptoms of UTI.

- Lab criteria: Negative urine culture (less than $10^{5}$ colony-forming-units $(\mathrm{CFU}) / \mathrm{mL})$.

Group (3): non febrile healthy children as a control.

- This group includes non febrile children, with no evidence of inflammation-related disease requiring a medical examination or lab investigations.

- Lab criteria: Negative urine culture (less than $10^{5}$ colony-forming-units $(\mathrm{CFU}) / \mathrm{mL})$.

\subsection{Exclusion Criteria}

Patients with history of UTI within less than 3 months, antibiotics therapy in the previous $72 \mathrm{~h}$, patients with congenital anomalies in urinary system and immunosupressed patients were excluded.

For all patients in the three groups:

Full history was taken and lab tests including complete blood count, C-reactive protein (CRP), serum creatinine, and urine creatinine measurement were done.

Urine samples were collected from all patients either by midstream technique in toilet trained children or using adhesive urine bags in younger children [16].

Routine microscopic and biochemical urine analysis were performed for all collected urine samples.

Urine culture was performed for patients in all groups on cystine lactose electrolyte deficient (CLED) agar (Thermo Fisher Scientific-UK) plate according to method described before [16].

- Pyuria was defined as $\geq 5 \mathrm{WBCs} / \mathrm{HPF}$.

- Dipstick tests were performed on all collected urine samples for detection of proteinuria which defined as protein score $\geq 1$ ( 1 to 4 ), nitrite production which defined as positive or negative. Leukocyte esterase production: defined as positive or negative.

Isolated organisms were identified using routine microbiological methods and API $20 \mathrm{E}$ and $20 \mathrm{NE}$ (bioMérieux) according to the manufacture instructions [16]. 


\subsection{Urine YKL-40 and uNGAL Assay}

For uYKL-40 and uNGAL levels assay, urine samples collected for culture and routine urine analysis were used. Urinary samples were cleared by centrifugation. Urine YKL-40 values were measured using Human Chitinase 3-like 1 Quantikine ELISA Kit ( $\mathrm{R} \& \mathrm{D}$ systems) according to the manufacture instructions.

Normally, uYKL-40 in healthy subjects can be undetectable or its level is less than $200 \mathrm{pg} / \mathrm{ml}$ [17].

Measuring urinary NGAL levels: Urine NGAL levels were measured using Human Lipocalin-2/NGAL Quantikine ELISA Kit (R \& D systems) according to the manufacture instructions. Urine NGAL and YKL-40 values were expressed as $\mathrm{ng} / \mathrm{ml}$ and $\mathrm{pg} / \mathrm{mL}$.

To nullify the effect of urine dilution on markers concentration, urine levels of YKL-40 and NGAL were normalized to urine creatinine level and expressed as urine YKL-40 and urine NGAL to urine creatinine ratio; uYKL-40/Cr (pg/mg) and uNGAL/Cr (ng/mg) respectively.

The study was approved by the institutional review board at faculty of medicine Mansoura Univerisy (code number R.19.11.670). Informed consent was obtained from parents of all children included in the study.

\subsection{Statistical Analysis}

Statistical Package for the Social Sciences (SPSS) software for Windows 20.0 was used for statistical analyses. Descriptive statistics was described as mean, standard deviation (s.d.). Statistical difference between two groups was analyzed by Chi-square test $\left(\chi^{2}\right)$ for categorical variables and independent sample Student's t-tests for continuous variables. Kolmogorov-Smirnov test was used to assess normality of continuous variables. To evaluate the diagnostic value of urine YKL-40 and NGAL levels for UTI, the receiver operating (ROC) curve analysis was used. Calculated areas under the ROC curve (AUC), optimum cut-off values, sensitivity and specificity were calculated. Values of $\mathrm{P}<0.05$ were considered to be significant.

\section{Results}

This study enrolled 150 children in Mansoura university children hospital out patients clinics department in the period extending from February 2016 to march 2018. Children were classified into 3 groups febrile patients with UTI symptoms and positive urine culture, febrile patients without UTI cause as evidenced by negative urine culture and the control group includes children without fever and with negative urinary culture. Urine and blood samples were collected from all patients in the three groups. Lab tests including CRP, CBC, serum creatinine measurement, urine creatinine measurement, routine urine analysis and urine culture were performed. Demographic and lab data of patients and controls included in the study are shown in Table 1. No significant difference 
Table 1. Demographic and lab data of the study groups.

\begin{tabular}{|c|c|c|c|c|c|c|}
\hline & \multirow{2}{*}{$\begin{array}{l}\text { Group (1) } \\
\text { Febrile children } \\
\text { with UTI }\end{array}$} & \multirow{2}{*}{$\begin{array}{c}\text { Group (2) } \\
\text { Febrile children } \\
\text { without UTI }\end{array}$} & \multirow{2}{*}{$\begin{array}{c}\text { Group (3) } \\
\text { Control }\end{array}$} & \multicolumn{3}{|c|}{$P$ value } \\
\hline & & & & $\mathrm{P}^{\mathrm{a}}$ & $\mathbf{P}^{\mathrm{b}}$ & $\mathrm{P}^{\mathrm{c}}$ \\
\hline $\begin{array}{l}\text { Age (years) } \\
\text { mean } \pm \text { SD }\end{array}$ & $5.7 \pm 4.4$ & $5 \pm 4.2$ & $4.5 \pm 4$ & 0.39 & 0.15 & 0.57 \\
\hline $\begin{array}{c}\text { Sex (No \%) } \\
\text { Male: } \\
\text { female }\end{array}$ & $\begin{array}{l}15(30) \\
35(70)\end{array}$ & $\begin{array}{l}13(26) \\
37(74)\end{array}$ & $\begin{array}{l}20(40) \\
30(60)\end{array}$ & 0.66 & 0.17 & 0.29 \\
\hline $\begin{array}{l}\text { Wbcs count } \\
(\text { mean } \pm S D)\end{array}$ & $12.6 \pm 2.2$ & $13.4 \pm 2.4$ & $5.4 \pm 1.3$ & 0.1 & $<0.0001$ & $<0.0001$ \\
\hline $\begin{array}{l}\text { Serum creatinine } \\
\quad(\text { mean } \pm S D)\end{array}$ & $0.7 \pm 0.2$ & $0.8 \pm 0.1$ & $0.7 \pm 0.1$ & 0.7 & 0.4 & 0.2 \\
\hline $\begin{array}{c}\text { CRP } \\
(\text { mean } \pm \mathrm{SD})\end{array}$ & $11.4 \pm 6.9$ & $13.9 \pm 9.9$ & $2.4 \pm 0.7$ & 0.1 & 0.0001 & 0.0001 \\
\hline $\begin{array}{c}\text { Pus cells/HPF } \\
(\text { mean } \pm \text { SD) }\end{array}$ & $43.82 \pm 33.85$ & $9.1 \pm 6.1$ & $4.1 \pm 1.7$ & 0.0001 & 0.0001 & 0.8 \\
\hline \multicolumn{7}{|l|}{ Proteniuria } \\
\hline Positive: & $26(52)$ & $9(18)$ & 0 & \multirow{2}{*}{0.0004} & \multirow[t]{2}{*}{ NA } & \multirow[t]{2}{*}{ NA } \\
\hline Negative & $24(48)$ & $41(82)$ & 50 & & & \\
\hline \multicolumn{7}{|l|}{ Nitrite } \\
\hline Positive: & $29(58)$ & 0 & 0 & NA & NA & NA \\
\hline Negative & $21(42)$ & 50 & 50 & & & \\
\hline
\end{tabular}

Wbcs: white blood cells; $\mathrm{P}^{\mathrm{a}}$ group 1 versus group 2; $\mathrm{P}^{\mathrm{b}}$ group 1 versus group 3; $\mathrm{P}^{\mathrm{c}}$ group 2 versus group 3 , NA: not applicable, SD: standard deviation.

regarding age and sex distribution between study groups.

Urine culture was done for all patients in the three groups. Significant bacteruria was detected in patients of group (1). Isolated bacteria were identified using standard microbiological methods, biochemical reactions and API $20 \mathrm{E}$ and API 20 NE. Types and percentages of isolated organisms are shown in Table 2. Escherichia coli represents the highest prevalence among organisms causing UTI in the first group (62\%). The $2^{\text {nd }}$ most common organism was Klebsiella (12\%). Patients in the $2^{\text {nd }}$ group were diagnosed gastero-enteritis, pneumonia, tonsillitis, and oteitis media.

Urine YKL-40 and uNGAL levels were measured in collected urine samples using ELISA kits according to the manufacture instructions. Normalized urine values of biomarkers to urinary creatinine level were estimated and were expressed as ng/mg for NGAL and pg/mg for YKL-40 Table 3. The mean uNGAL and UYKL-40 were significantly higher in febrile UTI group compared to the other two groups. Significant difference was detected regarding mean of uNGAL/Cr and uYKL-40/Cr in group (1). The mean values of uYKL-40, uNGAL, uNGAL/Cr and uYKL-40/Cr shows no significant difference between group (2) and group (3).

To compare the diagnostic value of both makers in UTI diagnosis, ROC curve analysis was used. The AUC, optimal cut-off value, sensitivity and specificity for uNGAL, uYKL-40, uNGAL/Cr and uYKL-40/Cr were estimated Table 4. 
Table 2. Results of urine culture in group (1).

\begin{tabular}{cc}
\hline Organism & Frequency NO (\%) \\
\hline E. coli & 31 \\
Klebsiella species & $(62)$ \\
Enterococci & 6 \\
& $(12)$ \\
Staphylococcus aureus & 5 \\
& $(10)$ \\
Proteus & 3 \\
& $(6)$ \\
Providencia & 1 \\
Enterobacter & $(2)$ \\
& 1 \\
& $(2)$ \\
\end{tabular}

E. coli represents the most common organism causing UTI in febrile UTI group.

Table 3. Urine levels of YKL-40, NGAL and the normalized values to urine creatinine level.

\begin{tabular}{|c|c|c|c|c|c|c|}
\hline & \multirow{2}{*}{$\begin{array}{l}\text { Group (1) Febrile } \\
\text { children with UTI }\end{array}$} & \multirow{2}{*}{$\begin{array}{c}\text { Group (2) Febrile } \\
\text { children without UTI }\end{array}$} & \multirow{2}{*}{ Group (3) Control } & \multicolumn{3}{|c|}{$P$ value } \\
\hline & & & & $\mathbf{P}^{\mathbf{a}}$ & $\mathrm{P}^{\mathrm{b}}$ & $P^{c}$ \\
\hline uYKL-40 (pg/ml) (mean \pm SD) & $980.2 \pm 2160$ & $151.2 \pm 54.5$ & $147.2 \pm 49.5$ & 0.008 & 0.009 & 0.7 \\
\hline $\mathrm{YKL}-40 / \mathrm{Cr}(\mathrm{pg} / \mathrm{mg})($ mean $\pm \mathrm{SD})$ & $661.7 \pm 1566$ & $134.1 \pm 91.1$ & $137.4 \pm 95.6$ & 0.02 & 0.02 & 0.8 \\
\hline uNGAL level $(\mathrm{ng} / \mathrm{ml})($ mean \pm SD) & $86.2 \pm 43.4$ & $30.6 \pm 16.5$ & $33.2 \pm 20.3$ & $<0.0001$ & $<0.0001$ & 0.5 \\
\hline $\mathrm{uNGAL} / \mathrm{Cr}(\mathrm{ng} / \mathrm{mg})($ mean $\pm \mathrm{SD})$ & $68.9 \pm 56.6$ & $28.2 \pm 23.3$ & $30 \pm 21.4$ & $<0.0001$ & $<0.0001$ & 0.7 \\
\hline
\end{tabular}

uYKL-40: urine YKL-40, YKL-40/Cr: urine YKL-40/ urine creatinine, uNGAL: urine NGAL, uNGAL/Cr: urine NGAL/urine creatinine; Student $t$ test was used to compare the values of markers and normalized markers; $\mathrm{P}^{\mathrm{a}}$ group 1 versus group2; $\mathrm{P}^{\mathrm{b}}$ group 1 versus group 3; $\mathrm{P}^{\mathrm{c}}$ group 2 versus group 3, SD: standard deviation.

Table 4. Area under the curve (AUC), optimal cut-off values, sensitivity and specificity for YKL-40, NGAL and their normalized values to urine creatinine level for the diagnosis of UTI in febrile children.

\begin{tabular}{cccccc}
\hline & Area under the curve (AUC) & P & Optimal cut-off value & Sensitivity (\%) & Specificity (\%) \\
\hline uYKL-40 (pg/ml) & 0.95 & $<0.0001$ & 171.5 & 84 & 82 \\
uYKL-40/Cr (pg/mg) & 0.81 & $<0.0001$ & 159.2 & 72 & 71 \\
u NGAL (ng/ml) & 0.90 & $<0.0001$ & 43.8 & 80 & 82 \\
uNGAL/Cr (ng/mg) & 0.75 & $<0.0001$ & 29 & 76 & 63 \\
\hline
\end{tabular}

uYKL-40: urine YKL-40, YKL-40/Cr: urine YKL-40/urine creatinine, uNGAL: urine NGAL, uNGAL/Cr: urine NGAL/urine creatinine.

The AUC of uYKL-40 was the highest value (0.95) for the diagnosis of UTI in febrile group with optimal cut off value $171.5 \mathrm{pg}$, followed by uYKL-40/Cr of 0.81 with best cut off value of $159.2 \mathrm{pg} / \mathrm{mg}$.

\section{Discussion}

The diagnosis of UTI in children is challenging. The absence of specific symp- 
toms, difficulty in mid stream urine collection for culture and analysis provide more obstacles for proper diagnosis [18] [19]. In addition, urine culture which represents the gold standard for diagnosis takes at least 2 days for confirmation of UTI [20]. The late diagnosis of UTI could result in severe complications such as formation of renal scar [21].

Treatment of UTI using empirical antibiotics can be started depending on abnormal finding in routine urine analysis as pyuria. In some cases, positive urine culture and pyuria may give false diagnosis of UTI. For example, pyuria in some viral infections and asymptomatic bacteriuria, this causes false prescrpation of unnecessary antibiotics [18] [22].

Therefore, more sensitive and specific diagnostic tests are required for better diagnosis of UTI in children. In case of UTI, activated neutrophil cells release NGAL in urine. So, urine NGAL is one of these markers that are assumed to be an accurate marker for diagnosis of UTI [4] [14] [23].

Mammalian chitinase-like proteins or YKL-40 is supposed to play role in defense mechanisms against many pathogens. YKL-40 has been indicated as a biomarker of sepsis [24].

Our study was aiming at evaluation of YKL-40 urine level as a marker for diagnosis of UTI in children. We compared the value of urine YKL-40 levels versus other marker for UTI diagnosis; NGAL. Both markers were measured in the three groups and the levels of both markers were normalized to urinary creatinine. The three groups of children included in the present study; febrile children due to UTI as indicted by positive urine culture, febrile children without UTI evidenced by negative urine culture, and control group without fever and with negative urine culture. Each group includes 50 patients or healthy controls.

Regarding the results of urine culture in febrile UTI group, Escherichia coli was the highest prevalence among bacterial agents causing UTI. It represents $(62 \%)$ of all positive cultures. Klebsiella spp were the $2^{\text {nd }}$ most common organism (12\%). These results match with other studies like Abdul Raheem et al., (2019) [25].

Our study determined the optimum cut-off value of uNGAL to be $43.8 \mathrm{ng} / \mathrm{ml}$ with sensitivity (80\%), specificity (82\%), and AUC (0.9). For uNGAL/Cr the optimal cut-off value was $(29 \mathrm{ng} / \mathrm{mg}$ ) with sensitivity $(76 \%)$, specificity $(63 \%)$, and AUC (0.75).

Our result matches with the result of Krzemien et al. (2018) [26]. They investigated the value of UNGAL in UTI in children. Their study determined optimum cut-off value $(42.2 \mathrm{ng} / \mathrm{ml})$ and AUC (0.76). However, they obtain lower sensitivity (73.8\%), specificity (72.2\%). Also, our result regarding uNGAL/Cr matches with results of Ylmaz et al. (2009) [2] they found optimum cut-off value $30 \mathrm{ng} / \mathrm{ml}$ for uNGAL/Cr but with higher sensitivity (98\%), specificity (76\%) and AUC (0.99).

However, our uNGAL cut-off value is higher than that of Yim et al. (2014) [5] (23.95 ng/mg) and for uNGAL/Cr (276.5), and Lee et al. (2015) [14] the optimial 
cut-off value was (108 $\mathrm{ng} / \mathrm{mg}$ ) with (97\%) sensitivity, (80.6\%) specificity and with similar AUC (0.91).

This discrepancy in the results may be due the difference in the age of the study groups or the inclusion of children with abnormal kidney functions in these studies. The children in our study have normal renal function and they didn't have history of congenital anomalies in urinary tract or recurrent UTI.

Our study revealed significant higher value of YKL-40 and YKL-40/Cr in UTI groups compared with sex and age matched febrile children due to other infections and control children without fever.

According to the ROC curve analysis, the optimal cut-off value of urineYKL-40 was (171.5 pg/ ml) with sensitivity (84\%), specificity (82\%), and AUC (0.95). The optimal cut-off value for urine YKL-40/Cr was $(159.2 \mathrm{pg} / \mathrm{mg})$ with sensitivity (72\%), specificity (71\%), and AUC (0.81). Our result agrees with the result of Kim et al., 2018 [27].

Up to our knowledge, this study is the first one to compare the value of urine YKL-40 versus urine NGAL value as biomarkers for diagnosing UTI in children.

According to our study uYKL-40 and uYKL-40/Cr values are of higher sensitivity and specificity than that of NGAL and UNGAL/Cr values in diagnosing febrile UTI. They can be used to differentiate UTI from other infections in febrile children.

Our study has some limitations. We did not investigate the effect of treatment on the level of urine YKL-40 and YKL-40/Cr and the possible use of this marker in the follow up and evaluation of the success of UTI treatment in children patients. Second, our study excludes patients with recurrent UTI and patients with congenital anomalies of urinary system. More studies are recommended to evaluate the use of YKL-40 as marker in these groups and the optimal cut-off value. In addition, the uses YKL-40 in treatment follow up.

\section{Conclusions}

The current study was designed to investigate the diagnostic value of urine YKL-40 level for early diagnosis of UTI in children. In addition, the study compared its value against value of urine NGAL level assay. The urine levels of these two markers were normalized to urine creatinine level. Up to our knowledge, this study is the first to compare the values of these two markers in UTI diagnosis in febrile children.

Our study reveals that uYKL- 40 and uYKL- $40 / C r$ can be used as a biomarker for diagnosis of simple uncomplicated UTI in febrile children. Urine YKL-40 has higher sensitivity and specificity compared to UNGAL level.

\section{Authors' Contributions Statement}

All authors have contributed equally to the study and manuscript.

\section{Acknowledgements}

The authors would like to thank all support staff and participating patients in 
this study.

\section{Funding}

None to declare.

\section{Conflicts of Interest}

The authors declare no conflicts of interest regarding the publication of this paper.

\section{References}

[1] Stein, R., Dogan, H.S., Hoebeke, P., Kočvara, R., Nijman, R.J., Radmayr, C., Tekgül, S., European Association of Urology and European Society for Pediatric Urology (2015) Urinary Tract Infection in Children: EAU/ESPU Guidelines. European Urology, 67, 546-558. https://doi.org/10.1016/j.eururo.2014.11.007

[2] Yilmaz, A., Sevketoglu, E., Gedikbasi, A., Karyagar, S., Kiyak, A., Mulazimoglu, M., Aydogan, G., Ozpacaci, T. and Hatipoglu, S. (2009) Early Prediction of Urinary Tract Infection with Urinary Neutrophil Gelatinase Associated Lipocalin. Pediatric Nephrology, 24, 2387-2392. https://doi.org/10.1007/s00467-009-1279-6

[3] Ghasemi, K., Esteghamati, M., Borzoo, S., Parvaneh, E. and Borzoo, S. (2016) Predictive Accuracy of Urinary Neutrophil Gelatinase Associated Lipocalin (NGAL) for Renal Parenchymal Involvement in Children with Acute Pyelonephritis. Electron Physician, 8, 1911-1917. https://doi.org/10.19082/1911

[4] Kim, B.H., Yu, N., Kim, H.R., Yun, K.W., Lim, I.S., Kim, T.H. and Lee, M.K. (2014) Evaluation of the Optimal Neutrophil Gelatinase-Associated Lipocalin Value as a Screening Biomarker for Urinary Tract Infections in Children. Annals of Laboratory Medicine, 34, 354-359. https://doi.org/10.3343/alm.2014.34.5.354

[5] Yim, H.E., Yim, H., Bae, E.S., Woo, S.U. and Yoo, K.H. (2014) Predictive Value of Urinary and Serum Biomarkers in Young Children with Febrile Urinary Tract Infections. Pediatric Nephrology, 29, 2181-2189. https://doi.org/10.1007/s00467-014-2845-0

[6] Johansen, J.S., Williamson, M.K., Rice, J.S. and Price, P.A. (1992) Identification of Proteins Secreted by Human Osteoblastic Cells in Culture. Journal of Bone and Mineral Research, 7, 501-512. https://doi.org/10.1002/jbmr.5650070506

[7] Johansen, J.S., Jensen, B.V., Roslind, A., Nielsen, D. and Price, P.A. (2006) Serum YKL-40, a New Prognostic Biomarker in Cancer Patients? Cancer Epidemiology, Biomarkers \& Prevention, 15, 194-202. https://doi.org/10.1158/1055-9965.EPI-05-0011

[8] Rehli, M., Niller, H.H., Ammon, C., Langmann, S., Schwarzfischer, L., Andreesen, R. and Krause, S.W. (2003) Transcriptional Regulation of CHI3L1, a Marker Gene for Late Stages of Macrophage Differentiation. The Journal of Biological Chemistry, 278, 44058-44067. https://doi.org/10.1074/jbc.M306792200

[9] Lee, C.G., Da Silva, C.A., Dela Cruz, C.S., Ahangari, F., Ma, B., Kang, M.J., He, C.H., Takyar, S. and Elias, J.A. (2011) Role of Chitin, Chitinase/Chitinase-Like Proteins in Inflammation, Tissue Remodeling, and Injury. Annual Review of Physiology, 73, 479-501. https://doi.org/10.1146/annurev-physiol-012110-142250

[10] Kazakova, M.H. and Sarafian, V.S. (2009) YKL-40: A Novel Biomarker in Clinical Practice? Folia Medica (Plovdiv), 51, 5-14. 
[11] Fantino, E., Gangell, C.L., Hartl, D., Sly, P.D. and Arest, C.F. (2014) Airway, But Not Serum or Urinary, Levels of YKL-40 Reflect Inflammation in Early Cystic Fibrosis Lung Disease. BMC Pulmonary Medicine, 14, Article No. 28. https://doi.org/10.1186/1471-2466-14-28

[12] Østergaard, C., Johansen, J.S., Benfield, T., Price, P.A. and Lundgren, J.D. (2002) YKL-40 Is Elevated in Cerebrospinal Fluid from Patients with Purulent Meningitis. Clinical and Diagnostic Laboratory Immunology, 9, 598-604. https://doi.org/10.1128/CDLI.9.3.598-604.2002

[13] Nasioudis, D. and Witkin, S.S. (2015) Neutrophil Gelatinase-Associated Lipocalin and Innate Immune Responses to Bacterial Infections. Medical Microbiology and Immunology, 204, 471-479. https://doi.org/10.1007/s00430-015-0394-1

[14] Lee, H.E., Kim, D.K., Kang, H.K., Park, K. (2015) The Diagnosis of Febrile Urinary Tract Infection in Children May Be Facilitated by Urinary Biomarkers. Pediatric Nephrology, 30, 123-130. https://doi.org/10.1007/s00467-014-2905-5

[15] Valdimarsson, S., Jodal, U., Barregård, L. and Hansson, S. (2017) Urine Neutrophil Gelatinase-Associated Lipocalin and Other Biomarkers in Infants with Urinary Tract Infection and in Febrile Controls. Pediatr Nephrol., 32, 2079-2087. https://doi.org/10.1007/s00467-017-3709-1

[16] Koneman, E.W., Allen, S.D., Janda, W.M., Schrecken-berger, R.C. and Winn, W.C. (1997) Introduction to Microbiology. Part II: Guidelines for the Collection Transport, Processing Analysis and Reporting of Culture from Specific Specimen Sources. In: Color Atlas and Textbook of Diagnostic Microbiology, 5th Edition, Lippincott-Raven, Philadelphia, 70-121.

[17] Lee, J.H., Kim, S.S., Kim, I.J., Song, S.H., Kim, Y.K., Kim, J., Jeon, Y.K., Kim, B.H. and Kwak, I.S. (2012) Clinical Implication of Plasma and Urine YKL-40, as a Proinflammatory Biomarker, on Early Stage of Nephropathy in Type 2 Diabetic Patients. Journal of Diabetes and Its Complications, 26, 308-312. https://doi.org/10.1016/j.jdiacomp.2012.04.012

[18] Tullus, K. (2011) Difficulties in Diagnosing Urinary Tract Infections in Small Children. Pediatric Nephrology, 26, 1923-1926. https://doi.org/10.1007/s00467-011-1966-y

[19] Lee, H.E., Lee, S.H., Baek, M., Choi, H. and Park, K. (2013) Urinary Measurement of Neutrophil Gelatinase Associated Lipocalin and Kidney Injury Molecule-1 Helps Diagnose Acute Pyelonephritis in a Preclinical Model. Journal of Biomarkers, 2013, Article ID: 413853. https://doi.org/10.1155/2013/413853

[20] Edefonti, A., Tel, F., Testa, S. and De Palma, D. (2014) Febrile Urinary Tract Infections: Clinical and Laboratory Diagnosis, Imaging, and Prognosis. Seminars in Nuclear Medicine, 44, 123-128. https://doi.org/10.1053/j.semnuclmed.2013.10.004

[21] Park, Y.S. (2012) Renal Scar Formation after Urinary Tract Infection in Children. Korean Journal of Pediatrics, 55, 367-370. https://doi.org/10.3345/kjp.2012.55.10.367

[22] Waseem, M., Chen, J., Paudel, G., Sharma, N., Castillo, M., Ain, Y. and Leber, M. (2014) Can a Simple Urinalysis Predict the Causative Agent and the Antibiotic Sensitivities? Pediatric Emergency Care, 30, 244-247. https://doi.org/10.1097/PEC.0000000000000105

[23] Ichino, M., Kuroyanagi, Y., Kusaka, M., Mori, T., Ishikawa, K., Shiroki, R., Kurahashi, H. and Hoshinaga, K. (2009) Increased Urinary Neutrophil Gelatinase Associated Lipocalin Levels in a Rat Model of Upper Urinary Tract Infection. Journal of Urology, 181, 2326-2331. https://doi.org/10.1016/j.juro.2009.01.010 
[24] Hattori, N., Oda, S., Sadahiro, T., Nakamura, M., Abe, R., Shinozaki, K., Nomura, F., Tomonaga, T., Matsushita, K., Kodera, Y., Sogawa, K., Satoh, M. and Hirasawa, H. (2009) YKL-40 Identified by Proteomic Analysis as a Biomarker of Sepsis. Shock, 32, 393-400. https://doi.org/10.1097/SHK.0b013e31819e2c0c

[25] Abdul Raheem, R.S., Hussein, M.A. and Al-Din, N.I. (2019) Causative Organism of Urinary Tract Infection and Drug Resistance in Children at Child's Central Teaching Hospital in Baghdad City. Journal of Pakistan Medical Association, 69, S59-S63.

[26] Krzemień, G., Pańczyk-Tomaszewska, M., Adamczuk, D., Kotuła, I., Demkow, U. and Szmigielska, A. (2018) Neutrophil Gelatinase-Associated Lipocalin: A Biomarker for Early Diagnosis of Urinary Tract Infections in Infants. Advances in EXperimental Medicine and Biology, 1047, 71-80.

https://doi.org/10.1007/5584 $2017 \quad 107$

[27] Kim, H.H., Chung, M.H., Bin, J.H., Cho, K.S., Lee, J., Suh, J.S. (2018) Urinary YKL-40 as a Candidate Biomarker for Febrile Urinary Tract Infection in Young Children. Annals of Laboratory Medicine, 38, 39-45.

https://doi.org/10.3343/alm.2018.38.1.39 